\title{
"FERRO, FAME AC PESTE OPPRESSA": L'AMMIRAGLIO BERNAT DE VILAMARÍ E IL BLOCCO NAVALE DI GENOVA (1456-1458)
}

\author{
ENRICO BASSO \\ Universitd di Genova (Italia)
}

Gli ultimi anni del sesto decennio del XV secolo furono sicuramente uno dei periodi più travagliati della storia di Genova medievale; ad Oriente, l'impero coloniale edificato nel corso di due secoli di continui sforzi andava sgretolandosi sotto i colpi dei Turchi: nel 1453 insieme a Costantinopoli anche Pera aveva dovuto capitolare di fronte alla Mezzalu$\mathrm{na}^{1}$, nel 1455 era stato il turno delle due Focee e delle loro preziosissime miniere di allume e da allora il pericolo non aveva cessato di gravare sulle residue posizioni di Chio e di Lesbo $^{2}$, mentre il malcontento e la rivolta serpeggiavano all'interno degli stabilimenti del $\mathrm{Mar} \mathrm{Nero}^{3}$, premuti anche all'esterno dalla minaccia tatara e dalle mire espansionistiche di alcune delle più giovani potenze dell'area pontica, come la Moldavia ${ }^{4}$. Ad Occidente la

'Si veda in proposito G.PISTARINo, Genovesi d'Oriente, "Civico Istituto Colombiano, Studi e Testi, serie storica a cura di Geo Pistarino" (S.T.), 14, Genova, 1990, pp. 281-382.

${ }^{2}$ Sugli eventi di questo periodo, ai vedano G. PISTARINO, Genovesi, cit., pp. 243-280 e 383-420; ID., I Signori del mare, S.T., 15, Genova, 1992, pp. 377464.

${ }^{3}$ Si veda A. M. CIPERIS, Vnutrennee polozenie $i$ klassovaja bor'ba v Kaffe v 50-70 8g. XV veka, in "UZ Turkmenskogo GU", 21 (1962), trad. it. a cura di A. PREFUMO, Situazione intema e lotta di classe a Caffa tra gli anni' 50 ' 70 del XV secolo, in AA.VV., Saggi e Documenti V: Storici Sovietici del Levante Genovese, S.T., 7, Genova, 1985, pp. 223-258.

"Su questo argomento si veda E. BASSO, "De rebus castri Ilicis et alia": Genova, la Valacchia e la Moldavia fra cooperazione e contrasto nel secondo Quattrocento. Relazione presentata al "Convegno italo-romeno 'Italia e Romania: due popoli e due storie a confronto', Venezia 6-10 marzo 1995", atti in corso di stampa.

"Anuario de Botudion Mediovalos", 24 (1994) 
situazione non era certo migliore, la rete delle rotte commerciali che costituivano il fondamento della vita economica genovese era infatti seriamente minacciata dall'affermazione della Corona d'Aragona nel Tirreno'; in parallelo all'espansione catalano-aragonese prima in Sicilia ed in Sardegna e poi nel Regno di Napoli, gli spazi per la libera navigazione genovese verso l'Africa, l'Oriente e la Penisola Iberica si erano andati gradualmente restringendo, e negli anni intorno alla metà del secolo era ormai divenuto palese come Alfonso V, che aveva identificato nella Repubblica ligure il principale ostacolo che ancora si frapponeva alla realizzazione del suo progetto imperiale, avesse ormai deciso di perseguire con ogni mezzo lo scopo di sottomettere o soffocare l'irriducibile avversaria ${ }^{6}$.

Visto il risultato fallimentare dei progetti intrapresi nella prima direzione, dapprima con l'appoggio offerto all'instaurazione ed al mantenimento della dominazione viscontea sulla citta ${ }^{7}$ e poi con l'imposizione su trono dogale di docili strumenti della politica aragonese quali i dogi Raffae$1 e^{8}$ e Barnaba Adorno 9 , e visto soprattutto il permanere della pericolosità dell'avversaria per la stabilità del suo impero, ampiamente provato dall'ap-

\footnotetext{
'Si vedano F. GIUNTA, Aragonesi e catalani nel Mediterraneo, I, Dal Regno al viceregno in Sicilia, Palermo, 1953; II, La presenza catalana nel Levante dalle origini a Giacomo II, Palermo, 1959; M. DEL TREPPO, I mercanti catalani e l'espansione della Corona aragonese nel secolo XV, Napoli, 1968; ID., L'espansione catalano-aragonese nel Mediterraneo, in Nuove questioni di storia medievale, Milano, 1969, pp. 259-300; G. PISTARINO, Espansione mediterranea della Corona d'Aragona, in "Segundo Congreso internacional de estudios sobre las culturas del Mediterraneo occidental", Barcelona 1975, Barcelona, 1978. Sulla rete delle rotte di navigazione genovesi nel Mediterraneo, si veda E. BASSO, Genova: un impero sul mare, Collana di Studi italo-iberici (CSII), 20, Cagliari, 1994, pp. 185-196.

${ }^{6}$ Sulla politica di Alfonso $\mathrm{V}$ come re di Napoli, si vedano E. DUPRÉ THESEIDER, La politica italiana di Alfonso il Magnanimo, Palma de Mallorca, 1955; E. PONTIERI, Alfonso il Magnanimo re di Napoli (1435-1458), Napoli, 1975; A. RYDER, The Kingdom of Naples under Alfonso the Magnanimous, Oxford, 1976.

'Su questo argomento si veda E. BASSO, Genova, cit., pp. 219- 261.

'Sul dogato di Raffaele Adomo (1442-1447) e sulla sua deposizione -favorita da Alfonsoad opera del cugino Bamaba nel 1447, si vedano L. LEVATI, Dogi perpetui della Repubblica di Genova (1338. 1528). Studio biografico, Genova, 1928, pp. 284-301; G. OlGIAT, Genova, 1446: la rivolta dei "patroni" contro il dogato di Raffaele Adomo, in "Nuova Rivista Storica", LXXII (1988), pp. 389- 464, in particolare pp. 443-444. Sull'umiliante trattato accettato da Raffaele nel 1444, in base al quale i Genovesi, oltre a riconoscere Alfonso come re di Napoli, si impegnavano alla consegna annuale di una patera aurea come simbolo di sottomissione, si veda G. OLGIATT, Classis contra regem Aragonum (Genova, 1453- 1454). Organizzazione militare ed economica della spedizione navale contro Napoli, CSII, 15, Cagliari, 1990, pp. 15-16. Il testo del trattato si trova in Archivio di Stato di Genova (ASG), Liber Jurium, III, cc. 45 r. -53 r.
}

${ }^{9}$ Sul dogato di Barmaba Adorno, si veda L. LEVAT1, Dogi perpetui, cit., pp. 302-314. 
poggio offerto da Genova tanto a Renato d'Angid ${ }^{10}$ quanto ad Antonio Centelles ed ai suoi compagni nei loro tentativi di rovesciare il governo aragonese a Napoli ${ }^{11}$, il Magnanimo -che oltretutto non aveva mai dimenticato l'umiliazione inferta dai Genovesi alla sua potenza e alla sua stessa persona nelle acque di Ponza ${ }^{12}$ - si era pero infine risolto ad impegnare direttamente le forze dei suoi regni nel tentativo di sconfiggere in modo definitivo la Repubblica per cancellarla dal novero delle Potenze del Mediterraneo ${ }^{13}$.

A questo fine, Alfonso intensifico non solo la sua abile azione diplomatica tendente ad isolare sempre più Genova nel quadro politico non solamente italiano, ma più in generale mediterraneo -che aveva avuto gia in precedenza episodi significativi con l'esclusione di Genova dalla pace di Lodi ${ }^{14}$ e soprattutto con le accuse rivolte ai Genovesi, i "Turchi italiani", di collaborazione militare con il Sultano nel Levante ${ }^{15}$-, ma anche la poli-

\footnotetext{
${ }^{10} \mathrm{Su}$ questo punto si veda G. OLGIA1T, La Repubblica di Genova nella guerra di successione al Regno di Napoli (1436-1442). Relazione presentata al "XIV Congresso di Storia della Corona d'Aragona, Sassari-Alghero 19-24 maggio 1990", atti in corso di stampa.

"Su questo argomento, cfr. G. OLGIATI, Classis contra regem Aragonum (Genova, 14531454). Organizzazione militare ed economica della spedizione navale contro Napoli, CSII, 15, Cagliari, 1990.

${ }^{12}$ Si vedano Georgii et lohannis Stellae Annales Genuenses, a cura di G. PETTI BALBI, "Rerum Italicarum Scriptores", XVII, parte II, Bologna, 1975, pp. 382-383; V. VITALE, Breviario della Storia di Genova (2 voll., Genova, 1955), I, pp. 156-157. L'ammiraglio della flotta genovese, il cancelliere Biagio Assereto, descrisse lo svolgimento della battaglia in una relazione inviata agli Anziani della Repubblica, cfr. G. BALBI, Uomini d'arme e di cultura nel Quattrocento genovese: Biagio Assereto, in "Atti della Società Ligure di Storia Patria" (ASLi), nuova serie II, fasc. Il, Genova, 1962, pp. 97-206, in particolare pp. 124-132; per quanto riguarda i prigionieri catturati sulle galee catalane, cfr. A. AGOSTO, Nuovi reperti archivistici sulla battaglia di Ponza, in "Mostra documentaria Liguria-Catalogna, XII-XV secolo", Genova, 1971, pp. 65-77.

${ }^{13}$ Sull'estremo tentativo messo in atto da Alfonso, in circostanze del tutto particolari quali erano quelle determinate dalla lotta per la successione al ducato di Milano scatenatasi alla morte di Filippo Maria Visconti, per legare Genova alla propria politica mediterranea, e sull'effimero ravvicinamento verificatosi, si veda G. OLGIATT, $L$ 'alleanza fallita: il trattato del 7 novembre 1447 tra Alfonso d'Aragona e Giano Campofregoso, in "La Storia dei Genovesi", X, Genova, 1990, pp. 319-368.

${ }^{14}$ Sulla situazione politica genovese in questo momento, si veda G. OLGIATI, Classis, cit., pp. 11-35 c, sull'esclusione del conflitto tra Genova ed Alfonso d'Aragona dai capitoli della pace di Lodi, pp. 213-214.

${ }^{15}$ Si vedano a questo proposito W. HEYD, Histoire du commerce du Levant au Moyen Age (2 voll., Leipzig, 1885-1886), II, pp. 305-307; G. PISTARINO, Genova e Barcellona: incontro e scontro di due civiltà, in "Atti del I Congresso storico Liguria-Catalogna", Bordighera, 1974, pp. 81-122, in particolare pp. 118-122; J. PAVIOT, Gênes et les Turcs (1444, 1453): sa défense contre les accusations d'une entente, in "La Storia dei Genovesi", IX, Genova, 1989,
} 
tica di armamento di navi corsare, alle quali era affidato l'incarico di tagliare le rotte di navigazione che collegavano Genova tanto con le sue superstiti colonie d'Oriente, quanto con i porti della Castiglia e dell'Africa, allo scopo di interrompere sia i contatti commerciali sui quali si reggeva in buona parte l'economia genovese, sia l'approvvigionamento di derrate alimentari -in particolare di grano africano- di fondamentale importanza per il vettovagliamento della popolazione della metropoli ligure ${ }^{16}$.

Nella primavera del 1456 , l'operazione di interruzione dei rifornimenti alimentari di Genova ad opera delle navi corsare catalane era ormai in pieno svolgimento, anche con l'appoggio più o meno palese degli stessi ufficiali del re, come dimostra l'episodio della cattura al largo di Oristano delle navi di Bartolomeo de Fronte e Francesco Bellesio, cariche di grano africano, da parte di galee che innalzavano le insegne del viceré di Sardegna, un'azione per la quale il doge e gli Anziani non si limitarono a protestare energicamente con il vicer ${ }^{17}$ e con lo stesso Alfonso ${ }^{18}$, ma si rivolsero addirittura al pontefice per denunciare la persistente ostilità della Corona d'Aragona nei confronti della Repubblica ${ }^{19}$; questa denuncia venne poi ripresa pochi mesi dopo, in toni ancor più energici ed accorati, nel testo di un'altra lettera ufficiale, datata 17 luglio, che venne questa volta inviata non solo al papa Callisto III, ma anche a Francesco Foscari doge di

pp. 129-137; G. OLGIATT, I Genovesi in Oriente dopo la caduta di Costantinopoli, in "Studi Balcanici", a cura di F. GUIDA e L. VALMARIN, Roma, 1989, pp. 45-59, in particolare p. 53; E. BASSO, Genoa and the Turks in the Balkan-Aegean area between diplomacy and espionnage. Relazione presentata al "International Congress Cultural, historical and ethnopolitical relations between Christiunity and Islam in the Balkans, XIV-XV centuries, Vama july 2-3 1994", atti in corso di stampa.

${ }^{16}$ Sul corsarismo catalano si vedano A. UNAL, Il "Libre de acordament". Arruolamenso di equipaggi calalani per la guerra di corsa nel'400, CSII, 4, Cagliari, 1982; P.F. SIMBULA, Corsari e pirati nei mari di Sardegna, CSII, 19, Cagliari, 1993. Sull'approvvigionamento di grano della piazza genovese, si veda quanto detto da D. GIOFFRE, Lettere di Giovanni da Pontremoli, mercante genovese (1453-1459), "Collana Storica di Fonti e Studi diretta da Geo Pistarino" (CSFS), 33, Genova, 1982, pp. XXXIII-XLII.

${ }^{17}$ ASG., Archivio Segreto (AS), Litteranum (Litt.), reg. 1785, c. 381 v. Ulteriori proteste per altri atti di pirateria commessi da patroni catalani vennero presentate al viceré il 7 giugno dello stesso anno; ASG, AS, Litt., reg. 1794, c. 743 v.

${ }^{18}$ ASG, AS, Litt., reg. 1785, c. 381 r.

${ }^{19}$ ASG, AS, Litt., reg. 1785 , c. 384 r. 
Venezia, a Francesco Sforza duca di Milano, alla Signoria di Firenze, ai re di Castiglia, Inghilterra e Francia, al duca di Borgogna ed all'imperatore Federico III, per ribadire di fronte a tutte le principali Potenze della Cristianità come la guerra non dichiarata condotta da Alfonso V contro Genova per mezzo dei suoi corsari non si limitasse a danneggiare economicamente la Repubblica, ma impedisse di fatto ai suoi governanti di intraprendere qualunque seria azione militare o diplomatica contro il dilagare dei Turchi nel Levante, a dispetto di tutti i progetti che erano stati dibattuti in merito $^{20}$.

Le proteste diplomatiche, per quanto energiche, non erano pero certamente in grado di far desistere il sovrano aragonese dal suo progetto; l'attività dei suoi corsari che incrociavano nelle acque fra la Liguria, la Corsica e la Provenza conobbe cosl un ulteriore incremento: una squadra di sette galee venne avvistata nel giugno $1456^{21}$, altre dieci comparvero in prossimità della stessa Genova nel dicembre dello stesso anno ${ }^{22}$, mentre altre ancora erano continuamente pronte a tendere agguati a quelle navi genovesi che avessero osato avventurarsi da sole in mare aperto ${ }^{23}$. Il doge Pietro Campofregoso ${ }^{24}$ ed il suo governo furono cosl costretti ad ordinare

\footnotetext{
${ }^{20} \mathrm{Il}$ giomo precedente anche i Maonesi di Chio e le logge dei mercanti genovesi in Siviglia e Londra erano stati avvertiti della rottura della tregua operata da Alfonso e dei problemi che questo fatto generava per i progetti di spedizione contro i Turchi; cfr. ASG, AS, Litt., reg. 1794 , cc. 761 v. -765 r. Sui numerosi progetti di crociata che in quel momento venivano discussi in Europa, si vedano N. JORGA, Notes et extraits pour senir d l'histoire des Croisades au XV siecle, 2 voll., Paris, 1902-1915; F. CARDINI, Le Crociate tra il mito e la storia, Roma, 1971, pp. 243-316; J. PAVIOT, Gênes et les Turcs, cit., p. 135; ID., Les navires du duc de Bourgogne Philippe le Bon (vers 1440-1465), in "Atti del V Convegno Internazionale di Studi Colombiani: Navi e navigazione nei secoli XV e XVI" (2 voll., Civico Istituto Colombiano, Genova, 1990), vol. I, pp. 167-195.

${ }^{21} \mathrm{Del}$ pericolo viene avvisato, il 15 giugno, Francesco Giustiniani de Garibaldo, in arrivo da Chio con la sua nave; ASG, AS, Litt., reg. 1794, cc. 749 r./v.

${ }^{22}$ Le galec erano state avvistate all'altezza di Portofino il 15 dicembre; ASG, AS, Litt., reg. 1794 , c. $814 \mathrm{v}$.

${ }^{23}$ Per questo vennero inviate navi in direzione della Provenza per scortare $\mathrm{i}$ carichi di grano, come ad esempio quella di Angelo Giovanni Lomellino il 29 dicembre; ASG, AS, Litt., reg. 1794 , c. 819 r. Il Lomellino, che era stato l'ultimo podestà genovese di Pera, svolse un'intensa attività di scorta alle navi onerarie in questo periodo, trovando infine la morte in uno scontro con pirati catalani nelle acque provenzali; si veda G. OLGIATI, Angelo Giovanni Lomellino: attività politica e mercantile dell'ultimo podestà di Pera, in "La Storia dei Genovesi", IX, cit., pp. 139-196, in particolare pp. 170-173.

${ }^{24}$ Sull'azione politica di Pietro Campofregoso, doge di Genova dal 1450 al 1458, si vedano L. LEVAT, Dogi perpetui, cit., pp. 355-379; A. BORLANDI, Ragione politica e ragione di famiglia nel dogato di Pietro Fregoso, in "La Storia dei Genovesi", IV, Genova, 1984, pp. 353-402; G. OLGIATT, Classis, cit., pp. 18-215.
} 
ai patroni di non avvicinarsi al Mar Ligure se non in convoglio ${ }^{25}$ e ad armare in tutta fretta le navi che si trovavano nel porto di Genova per poterle inviare incontro ai convogli come scorta ${ }^{26}$, un compito per il quale non si esito ad assoldare anche un notorio predone del mare come il pirata Battista Aicardo di Porto Maurizio, detto "Scarincio", nell'evidente intento di ripagare i catalani con la loro stessa moneta ${ }^{27}$. Questi provvedimenti, pero, non erano evidentemente sufficienti per allontanare da Genova, intorno alla quale si andava progressivamente stringendo dalla parte di terra l'accerchiamento delle forze dei fuoriusciti guidati dagli Adorno e sostenuti dallo stesso Magnanimo, lo spettro della carestia, come rivelano le preoccupazioni del governo nei confronti della sicurezza delle navi in arrivo dalla Provenza con carichi di grano $^{28}$ ed il pressante invito rivolto dal doge alla potente loggia dei mercanti genovesi a Siviglia affinché provvedesse a dirottare le navi cariche di grano in arrivo dall'Inghilterra e dirette in Africa verso il porto di Genova ${ }^{29}$.

La conquista di Portofino da parte dei fuoriusciti e dei loro alleati catalani forniva pero alla flotta corsara una base nelle immediate vicinanze dello stesso porto di Genova e rendeva sempre più concreta la minaccia di un attacco dal mare contro la città; venne pertanto proposto di ordinare alle navi di ritorno dalla Castiglia di unirsi a quelle in arrivo dall'Inghilterra e dalle Fiandre per muovere verso Genova in un unico, imponente convoglio, mentre altre due navi armate, quelle di Domenico Dentuto e di Angelo Giovanni Lomellino, venivano inviate incontro al convoglio in arrivo da Chio, che già aveva perduto la nave Doria, per scortarlo fino in Patria ${ }^{30}$, ed una terza, la Salvaiga, riceveva l'ordine di salpare alla volta della Castiglia con il preciso incarico di sorvegliare l'estuario del Guadalquivir allo scopo di prevenire che altre navi genovesi vi subissero la sorte di quella di

\footnotetext{
${ }^{25}$ ASG, AS, Litt., reg. 1794, c. 779 v.

${ }^{26}$ Si veda G. OlGiAT1, Angelo Giovanni Lomellino, cit., p. 171.

${ }^{27}$ Sulla figura di Scarincio, si vedano G. PISTARINO, Scarincio, corsaro ligure del Quattrocento, in "Liguria", XXXV-10, ottobre 1968; L. BALETTO, Battista Aicardo di Porto Maurizio, detto Scarincio, corsaro-pirata del secondo Quattrocento, in "Corsari turchi e barbareschi in Liguria. Atti del $1^{\circ}$ Convegno di studi (Ceriale, 7-8 giugno 1986)", Albenga, 1987, pp. 143-170.

${ }^{28}$ Sull'aumento dei prezzi del grano in Genova provocato dall'attività dei corsari catalani, si veda D. GIOFFRE, Lettere, cit., doc. 115, pp. 156-159, lettera del 1 agosto 1457.

${ }^{29}$ ASG, AS, Litt., reg. 1794 , cc. 812 v. -813 r.

${ }^{30}$ ASG, AS, Diversorum (Div.), reg. 560, cc. 14 r./v.
} 
Galeotto Pinello, catturata dai catalani quando già si riteneva al sicuro in acque castigliane ${ }^{31}$. Pochi giorni dopo, in una lettera inviata al papa il 21 marzo del $1457^{32}$ per ribadire le accuse contro Alfonso e la sua politica, venne per la prima volta dichiarato esplicitamente che la perdurante ostilità catalana aveva posto Genova in una situazione talmente disperata che non si escludeva che la Repubblica potesse cercare rifugio all'ombra delle bandiere del re di Francia Carlo VII, un'affermazione che, per quanto coincidesse con quanto in seguito avvenne realmente, aveva probabilmente in quellu specifico momento lo scopo di prospettare al pontefice ed agli altri principi italiani la possibilita che un ingresso del sovrano francese sulla scena politica italiana potesse compromettere i delicati equilibri diplomatici faticosamente raggiunti a Lodi pochi anni prima, riaccendendo ad esempio la devastante contesa per la successione al trono di Napoli.

La sottomissione alla Francia prospettata nella lettera sopra citata era pero in quel momento considerata da Pietro Campofregoso e dal governo genovese ancora come una sorta di extrema ratio, prima di giungere alla quale venne deciso di impegnare le estreme risorse della Repubblica nell'orrganizzazione di una risposta militare all'offensiva catalana, in primo luogo attraverso la costruzione di una flotta di galee che, sotto il comando di Tommasino Campofregoso, avrebbe dovuto strappare Portofino ai ribelli ed ai loro alleati ${ }^{33}$, ed in seguito con l'istituzione di uno specifico Officium, incaricato di adottare i provvedimenti per la sicurezza della navigazione e di reperire $\mathrm{i}$ fondi necessari per il loro finanziamento ${ }^{34}$, mentre al doge ed all'Officium Balie maritime veniva delegata tutta l'autorità sulla materia catalanica ${ }^{35}$. Contemporaneamente continuavano gli assoldamenti di esperti capitani di mare disposti a mettersi al sevizio di Genova con le loro navi per proteggere le rotte commerciali e soprattutto i vitali rifornimenti di derrate alimentari; ovviamente, in una situazione quale quella in cui si trovavano, i governanti genovesi erano costretti a guardare più alle capacità marinare di questi comandanti che alle loro qualità morali, cosic-

${ }^{31}$ ASG, AS, Div., reg. 560 , c. 17 v.

${ }^{32}$ ASG, AS, Litt., reg. 1794 , cc. 843 v. -844 r.

${ }^{33}$ ASG, AS, Div., reg. 560, cc. 26 r.-v.

${ }^{3}$ ASG, AS, Div., reg. 560 , c. 37 v. E' interessante notare come, pochi giomi dopo questo provvedimento, venne deciso di sottoporre a censura la corrispondenza dei mencanti catalani ancora residenti a Genova; ASG, AS, Div., reg. 560, c. 38 v.

${ }^{35}$ ASG, AS, Div., reg. 560 , cc. 39 r./v. 
ché, oltre al già ricordato Scarincio, vennero presi al servizio della Repubblica anche numerosi pirati, il più famoso dei quali fu sicuramente Giuliano Gattilusio $^{36}$, esponente del ramo cadetto della famiglia dei potenti signori greco-genovesi dell'isola di Lesbos $^{37}$, la comparsa del quale nelle acque liguri -a dispetto dei problemi che già in precedenza aveva creato alle autorità genovesi con la sua condotta piratesca ${ }^{38}$ - era stata salutata con

\footnotetext{
${ }^{36}$ Giuliano Gattilusio e ricordato fra i grandi pirati dell'epoca, insieme a Scarincio e ad altri, anche dal cronista fiorentino Benedetto Dei, a lungo agente dei Medici alla corte ottomana; si veda BENEDETTO DE, La Cronica dall 'anno 1400 all'anno 1500, a cura di $R$. BARDUCCI, Istituto per la storia degli antichi Stati italiani, Fonti e Studi, 1, Firenze, 1985, p. 125. Sulla figura di Giuliano Gattilusio, si vedano J. HEERS, Les Génois en Angleterre: la crise de 1458-1466, in AA.VV., "Studi in onore di Armando Sapori" (3 voll., Milano, 1957), II, pp. 807-832; ID., Gênes au XVe siecle, Paris, 1961, pp. 306-307; G. PISTARINO, I Signori, cit., Pp. 333-347; ID., Giuliano Gattilusio corsaro e pirata greco-genovese del secolo XV, in "Miscellanea Storica", vol. I, "Biblioteca dell'Accademia Olubrense", 12, Pietrabissara, 1992, pp. 63-77; E. BASSO, Pirati e pirateria nel Mediterraneo medievale: il caso di Giuliano Gattilusio. Relazione presentata all'"Intemational Congress The Gattilusii of Lesbos, Mythilene september 9-11, 1994", atti in corso di stampa.

${ }^{37}$ Sull'argomento e sulle vicende della famiglia, si vedano: A. LUXORO-G. PINELU GENTILE, Documenti riguardanti alcuni dinasti dell'Egeo, in "Giomale ligustico di archeologia, storia e belle arti", I (1874), pp. 81-90, 217-221; II (1875), pp. 86-93, 292- 297; III (1876), pp. 313-316; V (1878), pp. 345-372; W. MILLER, The Genoese colonies in Greece, in "Essays on the Latin Orient", Amsterdam, 1964, pp. 296-298; ID., The Gatrilusi of Lesbos (1355-1462), ibidem, pp. 313-353; G. PISTARINO, Genovesi, cit., pp. 383-420; G. OLGIAT, I Gattilusio, in "Dibattito su famiglie nobili del mondo coloniale genovese nel Levante. Atti del Convegno di Montoggio, 23 ottobre 1993", Accademia Ligure di Scienze e Lettere, Monografie, IX, Genova, 1994, pp. 85-99; G. PISTARINO, I Gattilusio di Mitilene, Signori dell'Egeo. Relazione presentata al]' "Intemational Congress 'The Gattilusi of Lesbos' ", cit.

${ }^{38}$ Il Gattilusio, oltre a navi cipriote e catalane, aveva assalito nell'Egeo anche la nave Cattanea, patronizata da Lorenzo Spinola; si veda G. PISTARINO, Giuliano Gattilusio, cit., pp. 67-68. L'attacco, avvenuto il 3 agosto 1456, venne denunciato dallo Spinola in un atto rogato a Chio dal notaio Tommaso Recco il 3 novembre 1456 (ASG, Notai, filza 848, doc. LXXXXVI/2), che mi è stato segnalato dalla cortesia della professoressa Laura Balletto, che qui ringrazio. La controversia con Paride Cattaneo derivata da questo atto di pirateria venne sottoposta ad arbitrato il 12 maggio 1457 (ASG, AS, Diversonum Communis Janue, busta $712 \mathrm{~b}$, doc. 3; documento segnalatomi dalla cortesia di Catherine Otten-Froux, che qui ringrazio), ma, nonostante il giudizio a lui favorevole, il Cattaneo attendeva ancora nel 1467 di essere rimborsato dal Gattilusio dei danni subiti, valutati nella cifra di 4.500 dictati; ASG, AS, Diversorum Communis Janue, filza 3049. Il Gattilusio aveva inoltre creato un nuovo problema al govemo genovese, proprio nel 1457 , con la cattura di un'imbarcazione carica di grano di proprietà del Signore di Piombino; a questo proposito, si veda ASG, AS, Litterarum, reg. 1794, cc. 851 v., 854 r., 855 r., 857 r., 862 r.; questi documenti sono stati editi da A. LUXORO-G. PINEILI GENTILE, Documenti, cit., fasc. V, doce. 40, 44-47, pp. 352-353, 355357, ma, come del resto in molte altre occasioni, l'opera dei due eruditi ottocenteschi a risultata, ad un controllo accurato, imprecisa ed inattendibile; oltre ad avere un carattere puramente, e discutibilmente, antologico, essa d infatti soggetta a clamorosi errori, come quello occorso appunto nel caso del primo dei documenti qui citati, nel quale la datazione della lettera, chiaramente espressa in calce al manoscritto, de stata riportata inspiegabilmente al 1452 , avvenimento del resto non infrequente -come si z detto- nel corpo di quest'opera, nella quale
} 
soddisfazione in considerazione dei servigi che si pensava avrebbe potuto rendere $^{39}$. Il principe-pirata venne dunque inviato ad incrociare nelle acque provenzali ed iberiche con l'autorizzazione di catturare e depredare tutte le navi catalane che gli fosse capitato di incontrare ${ }^{40}$, ma, a dimostrazione del rischio insito nel tentativo di valersi dei servigi di questi predoni del mare, anch'egli, come già il suo collega Scarincio ${ }^{41}$, approfitto troppo largamente della protezione concessagli dalle patenti che gli erano state rilasciate dal governo genovese catturando, oltre ad alcuni carichi di grano, anche una nave castigliana, un episodio dal quale scaturl per il governo una nuova complicazione diplomatica, assolutamente indesiderabile in quel particolare momento ${ }^{42}$.

Il sostanziale fallimento del tentativo di reazione contro l'offensiva catalana è testimoniato dai numerosi provvedimenti che le autorità genovesi dovettero adottare nel marzo 1458 nel tentativo di tutelare le sempre meno numerose navi che tentavano di forzare il blocco per raggiungere le loro destinazioni in Oriente 0 in Occidente ${ }^{43}$. Ad aggravare questa situazione già di per sé drammatica giunse, il 22 dello stesso mese, la notizia trasmes-

anche altri documenti sono stati oggetto di grossolani errori di datazione e di interpretazione.

39.ASG, AS, Litterarum, reg. 1794, c. 851 v.

${ }^{40}$ Il provvedimento venne adottato nel corso di un Gran Consiglio convocato il 6 luglio 1457, dopo l'arrivo della notizia della cattura della nave Lomellina; si veda ASG, AS, Div., reg. 561 , cc. 17 r. -18 r. Il Gattilusio, come risulta dalle istruzioni date dal governo l'8 luglio 1457 a Bartolomeo Cafeca, commissario a bordo della sua nave, avrebbe dovuto spingeni fino a Cadice per prendere sotto scorta le navi genovesi, e avrebbe avuto il diritto di trattenere un quarto del bottino predato su navi catalane eventualmente catturate; cfr. ASG, AS, Diversorum Communis Janue, filza 3042, doc. 26. Per quanto riguarda l'ammontaro della ricompensa pagata al Gattilusio ed i metodi di raccolta del denaro, si veda ASG, AS., Div., reg. 560 , cc. 39 r./v.; Litt., reg. 1785 , c. 450 r.

${ }^{41}$ Nell'estate del 1457, Scarincio aveva assalito navi fiorentine, costringendo il govemo genovese a ribadire alla Signoria che il pirata era stato assoldato solo per combattere i catalani; ASG, AS, Litt., reg. 1785 , cc. 453 v. -454 r.

42Rappresentanti del govemo furono immediatamente inviati a Savona per convincere il Gattilusio a restituire la nave castigliana; ASG, AS, Litterarum, reg. 1794 , c. 892 v. (20 settembre 1457); edito in A. LUXORO-G. PINELJ] GENTILE, Documenti, cit., fasc. V, doc. 49, pp. 357-358. Un invito ad esercitare pressioni su di lui affinche liberasse la nave catturata veniva anche indirizzato al suo fideiussore, Bartolomeo Doria quondain lacobi; ASG, AS, Litterarum, reg. 1794 , c. 893 r. (20 settembre 1457).

${ }^{43}$ ASG, AS, Div., reg. 560 , cc. 121 r. -123 r. 
sa al Gran Consiglio dai membri dell'Officium sanitatis, istituito alcuni mesi prima, dell'evidenziarsi dei primi sintomi del diffondersi in città della pestilenza, favorito evidentemente dall'assedio e dalla carestia continuamente incombente ${ }^{44}$.

$\mathrm{Fu}$ in questo frangente che Pietro Campofregoso ed i suoi consiglieri si risolsero a mettere in atto il proposito annunciato al pontefice più di un anno prima ed intrapresero l'organizzazione dell'ambasceria che, sotto la guida dell'esperto giurista e diplomatico Battista de Goano, avrebbe dovuto contattare dapprima Giovanni d'Angio duca di Calabria, il principale avversario di Alfonso $\mathrm{V}$ nella contesa per il trono napoletano, e quindi re Carlo VII per trattare il passaggio di Genova sotto la sovranità dei gigli di Fran$\mathrm{cia}^{45}$.

Probabilmente informato di queste deliberazioni dalle sue spie in città, il Magnanimo decise di entrare direttamente in azione per sventare il pericolo che Genova potesse divenire la base per le ambizioni angioine di riscossa nel Mezzogiorno d'Italia; il compito di sferrare l'offensiva decisiva contro la rivale gia piegata dalla fame e dalla peste -a causa della quale le autorità avevano dovuto adottare provvedimenti quali il divieto di riunioni numerose, la sospensione della celebrazione dei processi ed il rilascio dei carcerati della Malapaga, sempre nella speranza di rallentare la diffusione del contagio ${ }^{46}$ )-, venne aftidato dal re a quello che era sicuramente il suo più esperto comandante navale: Bernat de Vilamarr.

Il Vilamari, che aveva già fama di essere uno dei più grandi corsari del suo tempo ${ }^{47}$, era considerato dagli stessi Genovesi, come è possibile rilevare dalle ammonizioni rivolte in proposito ai loro comandanti, come uno dei maggiori strateghi esistenti nel campo della guerra navale $e^{48}$; a lui Alfonso ordinò di stringere ancor di più l'assedio per mare e per terra

\footnotetext{
${ }^{4}$ ASG, AS, Div., reg. 560 , c. 125 r. Sulla diffusione e le conseguenze del morbo ci offrono un vivace quadro le lettere di Giovanni da Pontremoli; D. GIOFFrE, Lettere, cit., docc. $145,153,157$, pp. 209-210, 225-226, 234-237.

${ }^{45}$ ASG, AS, Div., reg. 560, cc. 127 v. -128 v. Le deliberazioni ufficiali relative al passaggio sotto la sovranità francesc furono adottate l'l ed il 2 marzo 1458; ASG, AS, Div., reg. 560 , cc. 250 r. -251 r.

${ }^{46}$ ASG, AS, Div., reg. 560 , cc. 129 v. -130 v.

${ }^{47} \mathrm{Si}$ veda quanto detto di lui in BENEDETTO DEj, La Cronica, cit., p. 125.

${ }^{48}$ ASG, AS, Litt., reg. 1797 , cc. 48 v. 49 r.
} 
intorno a Genova per piegare la resistenza della popolazione e costringerla alla sottomissione, a qualunque costo.

In obbedienza a tali ordini, la flotta catalano-aragonese si avvicino ancor di più all'imboccatura del porto di Genova, bloccando qualunque tentativo di entrarvi o uscirne, mentre le truppe dei ribelli avanzavano ancor più in direzione delle mura della citta ${ }^{49}$; la fine della resistenza appariva ormai imminente, quando si verifico un avvenimento destinato a modificare profondamente la situazione: il 10 maggio 1458, forzando i] blocco delle truppe ribelli, Giovanni d'Angio, duca di Calabria e pretendente al trono di Napoli, entro in Genova alla testa di un consistente numero di soldati e balestrieri francesi in qualità di nuovo luogotenente governatore della città per il re di Francia ${ }^{\text {so }}$.

Uno dei primi atti del nuovo governatore, appena un giorno dopo il suo insediamento, fu quello di inviare all'ammiraglio catalano il proprio araldo con una lettera con la quale gli comunicava ufficialmente che Genova era adesso sotto la sovranità del re di Francia e che pertanto, in virtù della pace intercorrente tra la Francia e la Corona d'Aragona, la flotta catalano-aragonese avrebbe dovuto ritirarsi e togliere qualunque sostegno ai ribelli, mentre la composizione di tutte le divergenze esistenti fra re Alfonso ed i Genovesi sarebbe stata affidata alla mediazione di re Carlo VII e dei suoi ministri ${ }^{51}$. Gli ordini che il Vilamarí aveva ricevuto dal suo re erano pero di tutt'altro tenore, e probabilmente contemplavano anche l'atteggiamento da assumere in caso di intervento francese; la risposta al messaggio del duca fu cosı indiretta, ma assai esplicita: un contingente di fanterie catalane venne sbarcato a ponente della città, a Sampierdarena, ed un altro a levante, a Quarto, mentre gli agenti dell'ammiraglio tentavano di sollevare la rivolta fra gli abitanti della Val Polcevera. Un simile atteggiamento, cosi contrario alle regole del codice cavalleresco, suscito lo sdegno di Giovanni d'Angio, che provvide ad informarne dettagliatamente re Carlo, al quale rivolse nel contempo la richiesta pressante di invio di aiuti in uomini e denaro, necessario a riattare le strutture portuali e ad armare navi

\footnotetext{
${ }^{49}$ La rivolta era guidata dagli esiliati Adorno, appoggiati, oltre che da Alfonso V, anche dai Fieschi e dal marchese del Carretto; si veda V. VITALE, Breviario, cit., vol. I, p. 161

${ }^{50}$ ASG, AS, Div., reg. 560, c. 130 v.; Litt., reg. 1797, c. 21 r.

${ }^{\text {s1 }}$ ASG, AS, Litt., reg. 1797, c. 21 r.
} 
da impegnare in battaglia contro il nemico ${ }^{52}$; tuttavia il duca, ligio alle sue convenzioni di aristocratico francese, volle offrire a Bernat de Vilamarí un'ultima possibilità di ottemperare alle regole del codice cavalleresco inviandogli un'altra lettera, il 23 maggio, con la quale, oltre a rinnovare le proteste per il blocco navale e per gli sbarchi di truppe catalane, lo invitava a chiarire le sue intenzioni nei confronti dei sudditi e delle terre del re di Francia entro il termine ultimativo di tre giorni, trascorso il quale Giovanni avrebbe considerato offesi tanto l'onore del re quanto il proprio ed avrebbe fatto quanto il codice cavalleresco gli imponeva per ottenere soddisfazione del torto subito ${ }^{53}$.

L'orgoglioso cartello di sfida del governatore francese non dovette pero impressionare più di tanto Bernat de Vilamari, avvezzo alla dura e pragmatica mentalità dei corsari e soprattutto sicuro dell'appoggio e dell'approvazione del suo re verso le sue azioni contro Genova, poiché dal punto di vista di Alfonso l'intervento francese nella contesa non aveva minimamente modificato i termini della questione, anzi, aveva semmai reso la città ligure un'avversaria potenzialmente ancor più pericolosa, in quanto possibile base per operazioni angioine contro Napoli. L'ammiraglio poteva inoltre contare su un appoggio sempre crescente fra i malcontenti del governo genovese: alle file dei ribelli erano infatti andati ad aggiungersi anche alcuni rami di grandi casate ghibelline come Spinola, Doria e del Carretto, che vedevano con sfavore l'entrata in Genova dei Francesi, tradizionali alleati delle casate guelfe loro rivali ${ }^{\text {s4 }}$.

Anche Giovanni d'Angio dovette prendere atto di questa situazione e, abbandonate le speranze di una composizione "cavalleresca" della situazione, iniziare a studiare un piano di guerra per giungere a spezzare il blocco che stava ormai strangolando la città ed i suoi abitanti. Venne cosi intrapresa l'organizzazione di un'operazione navale: mentre richieste d'aiuto in uomini e navi venivano indirizzate a re Renatoss, precisi ordini venivano fatti pervenire a Pietro Giustiniani de Campis, ex-podestà di Chio e comandante del convoglio in arrivo dal Levante, al quale venne ingiunto di

${ }^{52}$ ASG, AS, Litt., reg. 1797, cc. 26 v. -27 r.

${ }^{53}$ ASG, AS, Litt., reg. 1797, c. 28 v.

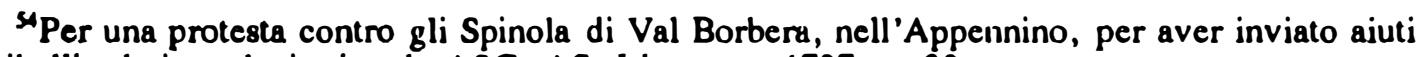
ai ribelli ed ai catalani, si veda ASG, AS, Litt., reg. 1797 , c. 30 r.

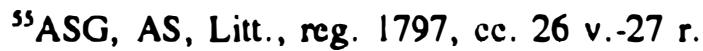


raccogliere tutte le sue navi nel porto corso di Bonifacio, dove avrebbe dovuto provvedere ad armarle per il combattimento ${ }^{56}$; per rinforzare ulteriormente la squadra del Giustiniani, che avrebbe dovuto attaccare di sorpresa $\mathrm{i}$ catalani nel momento in cui questi ultimi si sarebbero già trovati impegnati da una sortita di tutte le navi all'ancora nel porto di Genova, fu ordinato di raggiungerla anche alla grande nave di Oliviero Doria e Battista Paterio, in quel momento nel porto di Marsigliaa ${ }^{57}$, nonché alle unità che in quel momento si trovavano nel porto di Savona agli ordini di Giuliaino Gattilusio $^{58}$, il quale veniva cosl ancora una volta richiamato in servizio, nonostante i precedenti, proprio in considerazione del fatto che le sue caratteristiche lo rendevano adatto ad un piano di questo genere, come del resto lo stesso Scarincio, utilizzato anche in qualita di portaordini grazie alla sua abilità nello sgusciare fra le maglie del blocco navale ${ }^{59}$.

Il 14 giugno venne anche inviato al governatore della Corsica l'ordine di arruolare 400 o 500 uomini per rinforzare gli equipaggi della flot$t^{60}{ }^{60}$, e pochi giorni dopo, il 22 , furono fatte pervenire al Giustiniani istruzioni più dettagliate su cio che avrebbe dovuto fare una volta salpato alla volta di Genova, nonché su come avrebbe dovuto comportarsi nei confronti del Gattilusio ${ }^{61}$. Mentre si attendeva ansiosamente che, completati i preparativi, la flotta potesse passare all'azione, le truppe franco-genovesi erano impegnate nel contenimento delle puntate offensive delle fanterie catalane; fu proprio in quel momento, pero, che, come si puo rilevare da un dispaccio genovese del 28 giugno, le forze catalane assunsero un atteggiamento stranamente esitante, quasi come se il Vilamarf, fino a quel momento estremamente determinato nella sua condotta, fosse divenuto improvvisamente incerto su cio che doveva fare ${ }^{62}$.Il motivo del mutato atteggiamento dell'ammmiraglio, in quel momento ancora ignoto ai Genovesi ed al governatore, era l'improvvisa morte di re Alfonso, avvenuta la notte del 27 giugno, che evidentemente era stata tempestivamente comunicata al Vilamarr il qua-

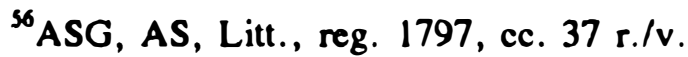

${ }^{57}$ ASG, AS, Litt., reg. 1797, cc. 43 v. 44 r.

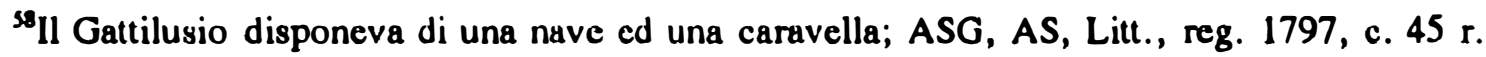

${ }^{39}$ ASG, AS, Litt., reg. 1797, c. 55 r.

${ }^{60}$ ASG, AS, Litt., reg. 1797, c. 44 v.

${ }^{61}$ ASG, AS, Litt., reg. 1797, cc. 48 v. 49 r.

${ }^{62}$ ASG, AS, Litt., reg. 1797, c. 50 v.
} 
le, in attesa che venisse definita la questione della successione al trono e che gli fossero fatti pervenire ordini dal nuovo sovrano, aveva evidentemente preferito sospendere ogni nuova iniziativa, limitandosi per il momento alla prosecuzione dell'assedio.

Gli assediati seppero approfittare dell'inattesa esitazione del nemico per cogliere alcuni successi, come la sortita che costrinse le truppe catalane che occupavano Sestri Ponente a reimbarcarsi il 30 giugno $^{63}$, ma furono colti completamente di sorpresa dall'improvvisa partenza della flotta catalana nella notte fra l' 1 ed il 2 luglio, tanto che, oltre ad esortare il castellano di Capodifaro alla vigilanza, nel caso si fosse trattato di una manovra diversiva $^{64}$, fu immediatamente inviato un corriere a Bonifacio, nel timore che il Vilamarí fosse venuto a sapere della flotta del Giustiniani ed avesse deciso di attaccarlo mentre le sue navi non erano ancora pronte alla battaglia $^{65}$. Fu soltanto il 5 luglio che, grazie ad un dispaccio inviato dalla Francia il giorno 2, il governatore e le altre autorità genovesi vennero finalmente informate della morte di Alfonso ${ }^{66}$ e poterono dunque avere un quadro più chiaro della situazione, che consentiva loro di comprendere le mosse del Vilamarf alla luce delle esigenze del nuovo sovrano di Napoli, Ferdinando d'Aragona, e di predisporre una nuova strategia contro i ribelli, rimasti abbandonati a se stessi. Mentre dunque venivano elaborati piani per recuperare le posizioni ancora sotto il controllo dei ribelli, come Portofino, veniva anche diramata, fra il 6 ed il 13 luglio, una serie di lettere rivolte alle comunità del Dominium alle quali, ora che le ostilità si erano di fatto risolte in un insperato successo per le forze franco-genovesi, veniva richiesto di prestare senza ulteriori indugi il dovuto atto di omaggio nei confronti del governo in carica ${ }^{67}$.

\footnotetext{
${ }^{63}$ ASG, AS, Litt., reg. 1797, c. 52 r.

${ }^{64}$ ASG, AS, Lill., reg. 1797, c. 53 r.

${ }^{65}$ ASG, AS, Litt., reg. 1797, c. 53 r.

${ }^{60}$ ASG, AS, Litt., reg. 1797, c. 55 r. In questa lettera, diretta a Pietro Giustiniani, viene anche specificata l'ora della inorte del re, le tre del mattino.

${ }^{67}$ ASG, AS, Lill., reg. 1797, ce. 55 v. -60 r.
} 
La ritirata della flotta catalano-aragonese provocd cosi il crollo delle forze ribelli in terraferma che, rimaste prive del loro principale sostegno, furono costrette a ritirarsi precipitosamente, limitandosi a mantenere alcune ben munite posizioni come Portofino ${ }^{68}$. Ancora una volta, Genova si era salvata quando ormai appariva sull'orlo del baratro e, anche se per la riconquista delle piazzaforti ancora tenute dai ribelli fu necessario organizzare nel corso dei mesi seguenti una poderosa spedizione militare $e^{69}$, il pericolo reale si era allontanato dalla Liguria insieme alle vele della flotta di Bernat de Vilamari, costretto ad abbandonare il campo quando era a un passo dal successo.

Anche se non era riuscito a raggiungere il suo obbiettivo finale, pero, il piano di Alfonso d'Aragona aveva comunque portato un profondo sconvolgimento nella vita interna di Genova: a parte i gravi problemi che il progressivo blocco navale aveva determinato nel campo economico, con la riduzione del volume dei traffici, in quello amministrativo, con la perdita del controllo su una parte del Dominium e le difficoltà nei contatti con le superstiti colonie orientali, ed in quello diplomatico, per l'impossibilità di coordinare insieme alle altre Potenze interessate una coerente risposta al dilagare della espansione ottomana, la conseguenza più evidente si era verificata nel campo politico con la perdita dell'indipendenza della Repubblica, ancora una volta costretta a sottomettersi ad un Signore straniero, sia pure di sua scelta ${ }^{70}$. Il fatto di aver dovuto nuovamente legare i suoi destini a quelli della Francia, ed in particolare a quelli della Casa d'Angio, comporto infatti per Genova negli anni successivi il coinvolgimento nella spedizione organizzata da Giovanni d'Angio per tentare di strappare a Ferdinando d'Aragona il trono di Napoli ${ }^{71}$, spedizione che, pur rispondendo in buona parte a quelli che erano anche gli interessi dei Genovesi, ebbe un prezzo politico, e soprattutto economico, difficilmente sostenibile per le

\footnotetext{
${ }^{68}$ Quasi contemporaneamente a quella di Alfonso si verifico anche la morte di Raffaele Adorno e di alcuni tra i principali capi della rivolta, lasciando i loro seguaci allo sbando; si veda G. OldilAT1, Classis, cit., p. 214.

${ }^{69}$ ASG, AS, Div., reg. 560, cc. 149 r. -167 r.

${ }^{70}$ Sul tentativo di Pictro Campolregoso di cacciare i Francesi da Genova e di riprendere il potere, conclusosi con la morte in combaltimento dell'ex-doge e del suo antico avversario, il conte Giovanni Filippo Fieschi, divenuto suo alleato, si vedano V. VITALE, Breviario, cit., I, p. 161; G. OlcilaT1, Classis, cil., p. 214.

"I resoconti delle spese e le liste degli arruolamenti per questa spedizione si trovano in ASG, AS, Div., reg. 560, ec. 168 v. -241 r.
} 
esauste casse genovesi, reso inoltre ancor più pesante dal fallimento finale dell'impresa che mise fine ad ogni speranza sia di trarne guadagno, sia, soprattutto, di poter cacciare la dinastia aragonese dal Mezzogiorno d'Ita$\mathrm{lia}^{2}$.

I problemi determinati dalla dominazione francese erano pero destinati ad essere transitori, mentre era definitiva, anche se sarebbero occorsi ancora alcuni anni perché i Genovesi se ne rendessero pienamente conto, la fine di quel pericolo rappresentato dall'espansione catalano-aragonese che aveva gravato sempre più minacciosamente su Genova nei lunghi decenni del regno di Alfonso il Magnanimo. Proprio l'improvvisa morte del re, e soprattutto le sue disposizioni testamentarie con le quali, attraverso la spartizione del suo impero tra il fratello ed il figlio illegittimo, egli stesso vanificava di fatto $i$ risultati di una politica perseguita con determinazione fin dagli inizi del suo regno, riapriva inaspettatamente la partita per il controllo delle rotte del Mediterraneo occidentale, che era sembrata ormai prossima a chiudersi con una definitiva vittoria della Corona d'Aragona, offrendo a Genova un'inattesa via d'uscita da una situazione politica ed economica difficilissima ${ }^{73}$.

La scomparsa del loro principale avversario, e la crisi nella quale sprofondo pochi anni dopo la Corona d'Aragona con lo scoppio della guerra civile, riaprivano dunque di fronte alle navi genovesi le vie del mare, quelle rotte commerciali che collegavano Genova ai porti di quella che stava ormai definitivamente affermandosi come la potenza egemone nella Penisola Iberica, la Castiglia, e grazie al controllo delle quali i Genovesi poterono non solo mettere in atto quel grandioso processo di riconversione economica da Oriente ad Occidente che consentl loro di rifarsi dei danni subiti nel Levante ad opera dei Turchi, ma anche gettare le basi per quello stretto legame politico ed economico con la Monarchia di Spagna che fu alla base del grandioso sviluppo del "Secolo dei genovesi"74.

\footnotetext{
${ }^{72}$ Per uno schematico resoconto degli avvenimenti di questi anni, si veda V. VITALE, Breviario, cit., I, pp. 160-161; molto più incisive sono però le pur brevi notazioni di G. OlgiaTt, Classis, cit., pp. 214-215.

${ }^{73}$ Sugli sviluppi della situazione politica e diplomatica, si vedano E. BASSO, Genova, cit., pp. 263-266; ID., Genoa and the Turks, cit.

${ }^{74}$ Su questo processo, si veda quanto detto da G. PISTARINo, I Signori, cit., pp. 377-464. Sul "secolo dei Genovesi", si veda A. PACINI, I presupposi politici del "secolo dei Genovesi". La riforma del 1528. ASLi, n.8., XXX (Gcnova, 1990), in particolare le pp. 7-48.
} 


\section{RÉSUMÉ}

Sur la base des documents inédites de l'Archive d'Etat de Gênes, l'Auteur donne une reconstruction des phases du siège naval de Gênes par la flotte catalano-aragonaise commandée par l'amiral Bernat de Vilamaŕ en 1456-1458, que représentait le dernier tentative du roi Alphonse V le Mag. nanime de detruire la Puissance rivale dans la lutte pour la maîtrise des routes de la Meditéranée occidentale.

\section{SUMMARY}

On the base of unpublished documents from the State Archive of Genoa, the Author retraces the phases of the naval blockade of Genoa by the Catalan-aragonese fleet led by admiral Bernat de Vilamars in 14561458, which represented the last attempt by king Alphonse $V$ the Magnanimous to destroy the rival Sea-power in the struggle for the control of Western Mediterranean commercial routes. 